OPEN

SUBJECT AREAS:

GENOME-WIDE ASSOCIATION STUDIES

STATISTICAL METHODS

Received

27 March 2014

Accepted

3 July 2014

Published

21 July 2014

Correspondence and requests for materials should be addressed to

Y.S. (yshen@c2b2.

columbia.edu)

* Current address:

Program in

Personalized \&

Genomic Medicine

and Department of

Medicine, University of

Maryland, Baltimore,

MD.

\section{Estimating heritability of drug-induced liver injury from common variants and implications for future study designs}

\author{
Casey Lynnette Overby ${ }^{1 *}$, George Hripcsak' \& Yufeng Shen ${ }^{1,2,3}$
}

\begin{abstract}
'Department of Biomedical Informatics, Columbia University, New York, NY, ${ }^{2}$ Department of Systems Biology, Columbia University, New York, NY, ${ }^{3}$ JP Sulzberger Columbia Genome Center, Columbia University, New York, NY.
\end{abstract}

Recent genome-wide association studies identified certain human leukocyote antigen (HLA) alleles as the major risk factors of drug-induced liver injuries (DILI). While these alleles often cause large relative risk, their predictive values are quite low due to low prevalence of idiosyncratic DILI. Finding additional risk factors is important for precision medicine. However, optimal design of further genetic studies is hindered by uncertain overall heritability of DILI. This is a common problem for low-prevalence pharmacological traits, since it is difficult to obtain clinical outcome data in families. Here we estimated the heritability $\left(h^{2}\right)$ of DILI from case-control genome-wide single nucleotide polymorphism data using a method based on random effect models. We estimated the proportion of $h^{2}$ captured by common SNPs for DILI to be between 0.3 and 0.5. For co-amoxiclav induced DILI, chromosome 6 explained part of the heritability, indicating additional contributions from common variants yet to be found. We performed simulations to assess the robustness of the $h^{2}$ estimate with limited sample size under low prevelance, a condition typical to studies on idiosyncratic pharmacological traits. Our findings suggest that common variants outside of HLA contribute to DILI susceptability; therefore, it is valuable to conduct further GWAS with expanded case collection.

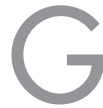

iven the relatively small sample sizes obtainable in pharmacogenetic studies ${ }^{1}$, consortia are forming with the goal of improving power to detect associations by pooling data generated from multiple studies. One effort relevant to this work is the International Serious Adverse Event Consortium (iSAEC). Phase 1 of the project has resulted in one of the largest DILI research collections in the world and initial studies have successfully identified genetic variants associated with DILI ${ }^{2}$.

While there are some successes identifying major risk factors of DILI (e.g., HLA-B*5701 as a major risk factor of liver injury due to flucloxacillin), no SNPs were identified in DILI across all drugs, nor have any been validated when flucloxacillin and coamoxiclav are excluded in this collection. This is likely due to limited power of detecting modest SNP effects with the relatively small cohort size. Understanding the heritability of these conditions is one step toward understanding genetic architecture of pharmacogenomic risk, and may provide valuable information for designing future studies.

Genome-wide association studies (GWAS) usually assume that common diseases are attributable to allelic variants present in more than $1-5 \%$ of the population ${ }^{3}$. However, most variants identified so far in GWAS analyses explain relatively small increases in risk. Also, in complex conditions where heritability has been estimated through twin and family studies, GWAS have failed to produce findings to support estimations. For example, the heritability of height is estimated to be $80 \%$, however only $4 \%$ can be explained by variants identified in GWAS studies". This discrepancy has been described as the missing heritability problem ${ }^{5}$. Proposed explanations for this issue are that rare variants with larger effects, gene-gene interactions, and/or gene-environment interactions are poorly detected by GWAS approaches ${ }^{6}$. Another explanation for the missing heritability is that common variants of smaller effect have not yet been identified. Peter Visscher's group has developed the Genome-wide Complex Trait Analysis (GCTA) method to measure the effect of common variants using a mixed model with genome-wide SNP data ${ }^{7}$. Applying this approach has provided further evidence that a substantial proportion of heritability is captured by common SNPs in complex traits including height and body mass index (BMI).

This approach is particularly attractive to apply in the context of pharmacogenomics studies, where ascertainment of family data is difficult or even impossible. Given the implicit assumption that adverse drug reactions 
Table 1 | Heritability estimates from iSAEC genome-wide data for co-amoxiclav and flucloxacilin induced drug-induced liver injury

\begin{tabular}{lcccccc} 
Drug (population) & Prevalence & No. cases & No. controls & Estimated $h_{\text {all }}^{2}$ (SE) & Estimated $h_{\text {chro }}^{2}$ (SE) & Estimated $h^{2}$ NOchro (SE) \\
\hline co-amoxiclav & 0.0005 & 201 & 532 & $0.40(0.16)$ & $0.17(0.041)$ & $0.38(0.17)$ \\
flucloxacillin & 0.0005 & 77 & 288 & $0.48(0.32)$ & $0.48(0.076)$ & $0.18(0.40)$ \\
co-amoxiclav & 0.0001 & 201 & 532 & $0.26(0.10)$ & $0.11(0.027)$ & $0.25(0.11)$ \\
flucloxacillin & 0.0001 & 77 & 288 & $0.31(0.21)$ & $0.31(0.05)$ & $0.12(0.26)$ \\
\hline
\end{tabular}

Table 2 | Heritability estimates from WTCCC genome-wide data for TID

\begin{tabular}{lccccc} 
TID Prevalence & No. cases & No. controls & Estimated $h^{2}{ }_{\text {all }}$ (SE) & Estimated $h_{\text {chro }}$ (SE) & Estimated $h^{2}{ }_{\text {Nochro }}$ (SE) \\
\hline 0.0005 & 1963 & 2938 & $0.26(0.022)$ & $0.068(0.008)$ & $0.22(0.022)$ \\
0.008 & 1963 & 2938 & $0.434(0.037)$ & $0.11(0.014)$ & $0.37(0.037)$ \\
\hline
\end{tabular}

(ADRs) are subject to substantial genetic control in pharmacogenomics studies, it is important to present evidence of the size of the genetic component (or heritability) for the trait under investigation.

Heritability estimates for complex traits are typically determined through twin studies. However, these approaches have limited utility in the context of susceptibility to ADRs ${ }^{8}$. Limitations are primarily due to difficulties recruiting and obtaining clinical outcome data in twins. This limitation might be overcome by applying the GCTA approach that estimates the effect of common variants from genome-wide SNP data. In this study we assessed the performance of this approach with DILI GWAS datasets.

\section{Results}

Proportion of heritability captured by genome-wide common variants for drug-induced liver injury. We estimated the proportion of $h^{2}$ captured by all genome-wide common SNPs, chromosome 6 genome-wide SNPs and genome-wide SNPs without chromosome 6 for DILI. Results for liver injuries induced by co-amoxiclav and flucloxacillin are summarized in Table 1 . We observe that for flucloxacillin induced DILI patients, chromosome 6 explained almost all of the heritability $\left(h^{2}\right.$ all $=0.48 \pm 0.313, h_{\text {chr6 }}^{2}=$ $\left.0.478 \pm 0.076, h_{\text {NOchr6 }}^{2}=0.18 \pm 0.40\right)$. Whereas with co-amoxiclav induced DILI patients, chromosome 6 explained part of the heritability $\left(h_{\text {all }}^{2}=0.40 \pm 0.16, h_{\text {chr6 }}^{2}=0.17 \pm 0.041, h_{\text {NOchr6 }}^{2}=\right.$ $0.378 \pm 0.17)$, indicating contributions from additional common variants are yet to be found. Estimates for $h^{2}$ may be underestimated due to GCTA algorithm constraints so that SNPheritability on the observed scale could not be greater than 1. Even so, this finding suggests that continuous collection of DILI cases is valuable for the potential of discovering additional associations with common genetic variants.

GCTA algorithm estimation of heritability for moderate sample sizes. The heritability of T1D is estimated from pedigree studies to be $0.9^{9,10}$. To date, the proportion of phenotypic variance explained by validated SNPs and genome-wide significant variants (including preGWAS loci with large effects) is $0.6^{9,11}$. In addition, the proportion of phenotypic variance explained when all GWAS SNPs are considered simultaneously is $0.3^{9,12}$. With the GCTA algorithm, we estimated the proportion of phenotypic variance explained by all genome-wide SNPs, chromosome 6 genome-wide SNPs, and genome-wide SNPs without chromosome 6. Results of data for all WTCCC data are summarized in Table 2. Results of data for 75 and 200 cases are summarized in Table 3. Our simulations to assess of the impact of sample sizes on heritability estimates indicated relatively stable estimates with moderate sample sizes. We estimate the proportion of $h^{2}$ captured by common SNPs for T1D to be, on average, $0.51 \pm$ 0.23 with a sample size of 75 and $0.46 \pm 0.15$ with a sample size of 200. These estimates are similar to estimations made with the entire dataset of 1963 cases and 2938 controls $\left(h^{2}{ }_{\text {all }}=0.44 \pm 0.037\right)$. Moreover, estimates calculated from sample sizes of 75 and 200 cases gave similar results, with both indicating that chromosome 6 explains part of the heritability (the $h_{c h r 6}^{2}$ estimate is $0.19 \pm 0.15$ with 75 cases and $0.12 \pm 0.058$ with 200 cases). For a sample size of 75, however, the standard deviation for our $h_{c h r}^{2}$ estimate is close to the mean. This indicates that calculations are not stable with smaller sample sizes, underscoring the uncertainty about flucloxacillininduced DILI. Estimates for $h_{c h r}^{2}$ calculated using this particular T1D dataset may also be underestimated due to inadequate SNP density in the MHC region in this particular data set.

Analysis of measurement errors in the drug-induced liver injury dataset. Lastly, we observe that the estimated $h^{2}$ is 0 for both flucloxacillin induced and co-amoxiclav induced DILI controls coded as cases. Results are summarized in Table 4. We conclude that there were no substantial measurement errors in our datasets.

\section{Discussion}

While further investigation is required to confirm the robustness of the GCTA algorithm for low prevalence traits such as DILI, this work highlights the potential value of its application. Here we were able to apply the algorithm to investigate the contribution of common variants on the chromosome level. Such investigations can provide insight into disease mechanism and into inter-individual variation. Moreover, for ADRs in particular, we are able to provide previously unobtainable estimates of heritability. Such estimates will help optimize designs of future studies for identifying additional genetic contributions to these conditions.

Findings suggest that collecting and genotyping more co-amoxiclav induced DILI cases is valuable for discovering additional associations. While further investigation is required to confirm the robustness of the GCTA algorithm for low prevalence traits like DILI, this work highlights the potential value of its application.

\begin{tabular}{|c|c|c|c|c|c|}
\hline TID Prevalence & No. Cases & No. Controls & Estimated $h_{\text {all }}^{2}(\mathrm{SD})$ & Estimated $h_{\text {chro }}^{2}(\mathrm{SD})$ & Estimated $h^{2}$ Nochro (SD) \\
\hline $\begin{array}{l}0.008 \\
0.008\end{array}$ & $\begin{array}{r}75 \\
200\end{array}$ & $\begin{array}{l}225 \\
600\end{array}$ & $\begin{array}{l}0.51(0.23) \\
0.46(0.18)\end{array}$ & $\begin{array}{l}0.19(0.15) \\
0.12(0.058)\end{array}$ & $\begin{array}{l}0.28(0.31) \\
0.35(0.16)\end{array}$ \\
\hline
\end{tabular}


Table 4 | Heritability estimation to assess measurement errors

DILI indicated drug (population)

No. cases

No. controls

Estimated $h^{2}$ all (SE)

flucloxacillin

co-amoxiclav

288 (controls coded as cases)

306 (controls coded as cases)

864 (WTCCC controls)

918 (WTCCC controls)

$0.0(0.11)$

$0.0(0.11)$

Particularly for pharmacological traits, we can provide previously unobtainable estimates of heritability. Such estimates will help optimize designs for future studies.

\section{Methods}

Study population. DILI datasets were from case-control studies of individuals taking flucloxacillin (77 cases and 288 population controls) ${ }^{2}$ and individuals taking coamoxiclav (201 cases and 532 population controls) ${ }^{13}$. The genotyping data were generated using the Illumina $1 \mathrm{M}$-duo described previously ${ }^{2,13}$.

Genome-wide complex trait analysis algorithm. The GCTA algorithm measures the effect of common variants with genome-wide SNP data using a mixed model approach $^{7}$. The algorithm involves first estimating the genetic relationship matrix (GRM) of all individuals, then fitting the GRM in a mixed linear model (MLM) for binary traits to estimate the proportion of variance explained by all the autosomal SNPs. We used GCTA algorithm extended for case-control designs ${ }^{14}$ to estimate $h^{2}$ captured by common SNPs for DILI. We also assessed the robustness of the GCTA algorithm with datasets of moderate sample sizes and low prevalence.

Estimating proportion of heritability captured by common variants. We used the GCTA algorithm to estimate $h^{2}$ from iSAEC DILI genome-wide data (all chromosomes), chromosome 6 data, and genome-wide data without chromosome 6 . We also estimated $h^{2}$ for all individuals (co-amoxiclav or flucloxacillin induced DILI), for individuals with co-amoxiclav induced DILI, and for individuals with flucloxacillin induced DILI. For individuals with co-amoxiclav induced DILI we evaluated northwest Europeans only and southern Europeans both together and separately. All analyses were performed separately. For each population and dataset, unless otherwise noted, we calculated $h^{2}$ adjusting for prediction errors due to global structure and local structure. We used principal components analysis (PCA) to adjust for prediction errors due to global structure. Two principal components were included as regression covariates in the mixed model. We also used an optional functionality of the GCTA tool to adjust for prediction errors due to imperfect linkage disequilibrium. All analyses were performed for DILI prevalence estimated to be $0.0001^{2,15}$ and 0.0005 . We reported main results using a prevalence of 0.0005 based on recent work (the estimated rate is 0.00043 in Iceland population ${ }^{16}$ ) and a general trend of underreporting of DILI incidences ${ }^{17}$.

Assessing the robustness of the GCTA algorithm with moderate sample sizes. To evaluate the robustness of the GCTA algorithm with datasets of moderate sample sizes, we conducted a positive control and negative control experiment. We performed simulations for our positive control experiment. Simulations involved estimating $h^{2}$ for subsets of cases and controls from a Type I Diabetes (T1D) dataset. We choose to use a T1D because the histocompatibility complex (MHC) region on chromosome 6 has major genetic contribution to risks of both $\mathrm{DILI}^{2}$ and T1D ${ }^{11,18}$. Specifically, we used the Welcome Trust Case Control Consortium (WTCCC) T1D dataset $\left(1963\right.$ cases and 2938 controls) ${ }^{18}$. Genotyping was conducted for the WTCCC study using an Illumina $550 \mathrm{~K}$ chip. We estimated $h^{2}$ from genome-wide data (all chromosomes), chromosome 6 data, and genome-wide data without chromosome 6 for cases and controls with a $1: 3$ ratio, where the number of cases were 75 and 200 to reflect sample sizes similar to iSAEC DILI sample sizes. Estimates for $h^{2}$ were averaged over twenty random selections of cases and controls for these sample sizes. The prevalence of T1D is estimated to be $0.008^{19}$. We report the mean and standard deviation of the twenty $h^{2}$ estimates. We also report $h^{2}$ estimates and standard errors with the full WTCCC T1D dataset and the GCTA parameter for prevalence set at 0.0005 and 0.008 . Given the WTCCC data were collected from a homogenous population, however, we do not adjust for prediction errors due to global structure. For a negative control experiment we tested for measurement errors by estimating $h^{2}$ for DILI controls coded as cases with WTCCC controls. This analysis was performed with northwestern European controls from the flucloxacillinin induced and co-amoxiclav induced DILI datasets. The prevalence of DILI was set to be 0.0005 .

1. Jorgensen, A. L. \& Williamson, P. R. Methodological quality of pharmacogenetic studies: issues of concern. Stat Med 27, 6547-6569, doi:10.1002/sim.3420 (2008).

2. Daly, A. K. et al. HLA-B*5701 genotype is a major determinant of drug-induced liver injury due to flucloxacillin. Nat Genet 41, 816-819, doi:10.1038/ng.379 (2009).

3. Collins, F. S., Guyer, M. S. \& Charkravarti, A. Variations on a theme: cataloging human DNA sequence variation. Science 278, 1580-1581 (1997).

4. McCarthy, M. I. et al. Genome-wide association studies for complex traits: consensus, uncertainty and challenges. Nat Rev Genet 9, 356-369, doi:10.1038/ nrg2344 (2008).
5. Maher, B. Personal genomes: The case of the missing heritability. Nature 456, 18-21, doi:10.1038/456018a (2008).

6. Manolio, T. A. et al. Finding the missing heritability of complex diseases. Nature 461, 747-753, doi:10.1038/nature08494 (2009).

7. Yang, J. et al. Common SNPs explain a large proportion of the heritability for human height. Nat Genet 42, 565-569, doi:10.1038/ng.608 (2010).

8. Malhotra, A. K., Murphy, G. M., Jr. \& Kennedy, J. L. Pharmacogenetics of psychotropic drug response. Am J Psychiatry 161, 780-796 (2004).

9. Visscher, P. M., Brown, M. A., McCarthy, M. I. \& Yang, J. Five years of GWAS discovery. Am J Hum Genet 90, 7-24, doi:10.1016/j.ajhg.2011.11.029 (2012).

10. Hyttinen, V., Kaprio, J., Kinnunen, L., Koskenvuo, M. \& Tuomilehto, J. Genetic liability of type 1 diabetes and the onset age among 22,650 young Finnish twin pairs: a nationwide follow-up study. Diabetes 52, 1052-1055 (2003).

11. Polychronakos, C. \& Li, Q. Understanding type 1 diabetes through genetics: advances and prospects. Nat Rev Genet 12, 781-792, doi:10.1038/nrg3069 (2011).

12. Anderson, C. A. et al. Meta-analysis identifies 29 additional ulcerative colitis risk loci, increasing the number of confirmed associations to 47. Nat Genet 43 , 246-252, doi:10.1038/ng.764 (2011).

13. Lucena, M. I. et al. Susceptibility to amoxicillin-clavulanate-induced liver injury is influenced by multiple HLA class I and II alleles. Gastroenterology 141, 338-347, doi:10.1053/j.gastro.2011.04.001 (2011).

14. Lee, S. H., Wray, N. R., Goddard, M. E. \& Visscher, P. M. Estimating missing heritability for disease from genome-wide association studies. Am J Hum Genet 88, 294-305, doi:10.1016/j.ajhg.2011.02.002 (2011).

15. Danan, G. \& Benichou, C. Causality assessment of adverse reactions to drugs--I. A novel method based on the conclusions of international consensus meetings: application to drug-induced liver injuries. J Clin Epidemiol 46, 1323-1330 (1993).

16. Bjornsson, E. S., Bergmann, O. M., Bjornsson, H. K., Kvaran, R. B. \& Olafsson, S. Incidence, presentation, and outcomes in patients with drug-induced liver injury in the general population of Iceland. Gastroenterology 144, 1419-1425, 1425 e1411-1413; quiz e1419-1420, doi:10.1053/j.gastro.2013.02.006 (2013).

17. Larrey, D. Epidemiology and individual susceptibility to adverse drug reactions affecting the liver. Seminars in liver disease 22, 145-155, doi:10.1055/s-200230105 (2002).

18. Nejentsev, S. et al. Localization of type 1 diabetes susceptibility to the MHC class I genes HLA-B and HLA-A. Nature 450, 887-892, doi:10.1038/nature06406 (2007).

19. Centers for Disease Control and Prevention, "CDC - Data Sources, References, and Methods - 2011 National Diabetes Fact Sheet - Publications - Diabetes DDT", $<$ http://www.cdc.gov/diabetes/pubs/references11.htm> (2011). (Date of access: $3 / 26 / 2014)$.

\section{Acknowledgments}

CLO and GH are supported by NIH NLM \#T15 LM007079. GH is also supported by NIH NLM R01 LM006910. YS is supported by Department of Systems Biology. This study makes use of data generated by the Wellcome Trust Case Control Consortium. A full list of the investigators who contributed to the generation of the data is available from www.wtccc.org. uk. This study also makes use of data generated by the International Serious Adverse Event Consortium (iSAEC). A full list of the investigators who contributed to the generation of the data is available from www.saeconsoritum.org.

\section{Author contributions}

C.L.O. and Y.S. designed the study, analyzed and interpreted the data, and wrote the manuscript. C.L.O. performed the experiments. G.H. interpreted the data and critically revised the manuscript for important content. All authors approved the final version of the manuscript.

\section{Additional information}

Competing financial interests: The authors declare no competing financial interests. How to cite this article: Overby, C.L., Hripcsak, G. \& Shen, Y. Estimating heritability of drug-induced liver injury from common variants and implications for future study designs. Sci. Rep. 4, 5762; DOI:10.1038/srep05762 (2014).

This work is licensed under a Creative Commons Attribution 4.0 International License. The images or other third party material in this article are included in the article's Creative Commons license, unless indicated otherwise in the credit line; if the material is not included under the Creative Commons license, users will need to obtain permission from the license holder in order to reproduce the material. To view a copy of this license, visit http://creativecommons.org/licenses/by/4.0/ 